\title{
White organic light-emitting devices by employing dual sub-monolayer
}

\author{
Lishuang $\mathrm{Wu}^{\mathrm{a}}$,Huishan Yang ${ }^{\mathrm{b} *}$ \\ College of Physics and Information Engineering, Quanzhou Normal University, Quanzhou 362000, \\ People's Republic of China \\ a email:lishuangw@126.com;
}

*Author to whom correspondence should be email addressed: ${ }^{b}$ yanghuishan1697@163.com

Keywords: White organic light-emitting devices; Dual sub-monolayer; Luminance; Efficiency; The Commission Internationale de 1'Eclairage (CIE) coordinates

Abstract. White organic light-emitting device have been fabricated by using highly blue fluorescent dye 9,10-di (2-naphthyl) anthracene(ADN) and 4- (dicyanomethylene) -2-t-butyle-6-(1,1,7,7tetramethyljulolidyl-9-enyl)-4H-pyran (DCJTB), together with well known green fluorescent dye 10(2-benzothiazolyl) -2,3,6,7-tetrahydro-1,1,7,7-tetramethyl-1H,5H,11H(1)-benzopyroyran-o (6,7-8-i,j) quinolizin-11-one(C545T). The fabrication of multilayer WOLEDs did not involve the hard-to-control doping process. The structure of the device is ITO/2T-NATA (50nm)/ AND (15 nm ) / C545T $(0.05 \mathrm{~nm}) / \mathrm{Alq} 3(5 \mathrm{~nm}) / \mathrm{DCJTB}(\mathrm{x} \mathrm{nm}) / \mathrm{Alq} 3(50 \mathrm{~nm}) / \mathrm{LiF}(0.5 \mathrm{~nm}) / \mathrm{Al}$, where $\mathrm{x}=0.025$ $\mathrm{nm}, 0.05 \mathrm{~nm}$ and $0.075 \mathrm{~nm}$, called device A-C, respectively. where 4,4'4'-Tris ( N-(2-naphthyl) -Nphenyl-amino)- triphenylamine (2T-NATA) act as hole transport layer, ADN act as blue emitting layer, DCJTB act as sub-monolayer red emitting layer, C545T act as sub-monolayer green emitting layer, tris- (8-hydroxyquinoline) aluminium $\left(\mathrm{Alq}_{3}\right)$ act as electron transport layer, WOLEDs of device $\mathrm{A}, \mathrm{B}$, and $\mathrm{C}$, different in layer thickness of DCJTB. To change the thickness of sub-monolayer DCJTB, WOLEDs was obtained. When $\mathrm{x}=0.025$, the Commission Internationale de 1'Eclairage (CIE) coordinates of the device A change from $(0.3212,0.2992)$ at $4 \mathrm{~V}$ to $(0.3018,0.3185)$ at $13 \mathrm{~V}$ that are well in the white region, respectively. Its maximum luminance was $8520 \mathrm{~cd} / \mathrm{m}^{2}$ at $13 \mathrm{~V}$, maximum current efficiency, maximum power efficiency was $9.92 \mathrm{~cd} / \mathrm{A}$ at $6 \mathrm{~V}, 2.09 \mathrm{~lm} / \mathrm{W}$ at $4 \mathrm{~V}$, respectively.

\section{Introduction}

Organic light-emitting diodes (OLEDs) are developed fast since the important work in 1987[1], and have shown their remarkable application in flat panel display and lighting nowadays [2-5].For the applications in display as well as solid-state lighting. Generally, white OLEDs (WOLEDs) can be obtained by employing two or more different complementary colors emitters in the emitting layer [6-9]. Consequently, the dopant light-emitter of guest-host system becomes a universal method for solving the quenching problem [10-15]. However, considering that the reproducibility of the optimum doping level requires careful manufacturing control and that the prolonged operation of the devices may lead to the phase separation of the dopant and host materials, doped OLEDs are relatively more difficult to adapt to practical applications than their nondoped counterparts. In this letter, the fabrication of multilayer WOLEDs did not involve the hard-to-control doping process. Sub -monolayer DCJTB act as red emitting layer, C545T act as green emitting layer, the effect of DCJTB emission on the WOLED is demonstrated. The simple device structures and excellent reproducibility make them well suited to low-cost lighting applications and beneficial to the industrialization.

\section{Experimental}

Fig. 1 shows the device structure of the OLEDs. The devices are prepared in a vacuum chamber at a pressure of $4.0^{*} 10^{-4}$ pa by thermal evaporation onto a cleaned indium tin oxide (ITO) coated glass substrate. The multilayer devices fabricated have the following structure: ITO/ 2T-NATA $(50 \mathrm{~nm}) /$ AND(15 nm )/C545T(0.05 nm)/Alq3(5 nm)/DCJTB (x nm)/ Alq3 (50 nm)/LiF (0.5nm)/Al, where $\mathrm{x}=0.025 \mathrm{~nm}, 0.05 \mathrm{~nm}, 0.075 \mathrm{~nm}$, called device A-C, respectively. The thickness of layers 
was controlled by quartz crystal monitor. The EL spectra and CIE coordination of the devices were measured by PR655 Spectra Scan spectrophotometer and the current-voltage-brightness characteristics were simultaneously measured by a voltage-current source (Keithley 2400). All the measurements were carried out at room temperature under ambient atmosphere.

\begin{tabular}{|c|}
\hline $\operatorname{LiF}(0.5 \mathrm{~nm}) / \mathrm{Al}(200 \mathrm{~nm})$ \\
\hline $\operatorname{Alq3}(50 \mathrm{~nm})$ \\
\hline $\operatorname{DCJTB}(\mathbf{x ~ n m})$ \\
\hline $\operatorname{Alq3}(5 \mathrm{~nm})$ \\
\hline $\operatorname{C545T}(0.05 \mathrm{~nm})$ \\
\hline $\operatorname{ADN}(15 \mathrm{~nm})$ \\
\hline 2T-NATA $(50 \mathrm{~nm})$ \\
\hline ITO \\
\hline
\end{tabular}

Fig. 1 The device structure of the OLEDs

\section{Results and discussion}

Fig.2 shows the Normalized EL intensity of the different devices at different voltage. The devices A-C emerging three main emission peaks at $458 \mathrm{~nm}, 504 \mathrm{~nm}, 612 \mathrm{~nm}$ originating from ADN, C545T, DCJTB,respectively. The device A shows quite strong blue emitting compared with the device B and $\mathrm{C}$, this observation is attributed to thin sub-monolayer from DCJTB, more excitons formed in AND.
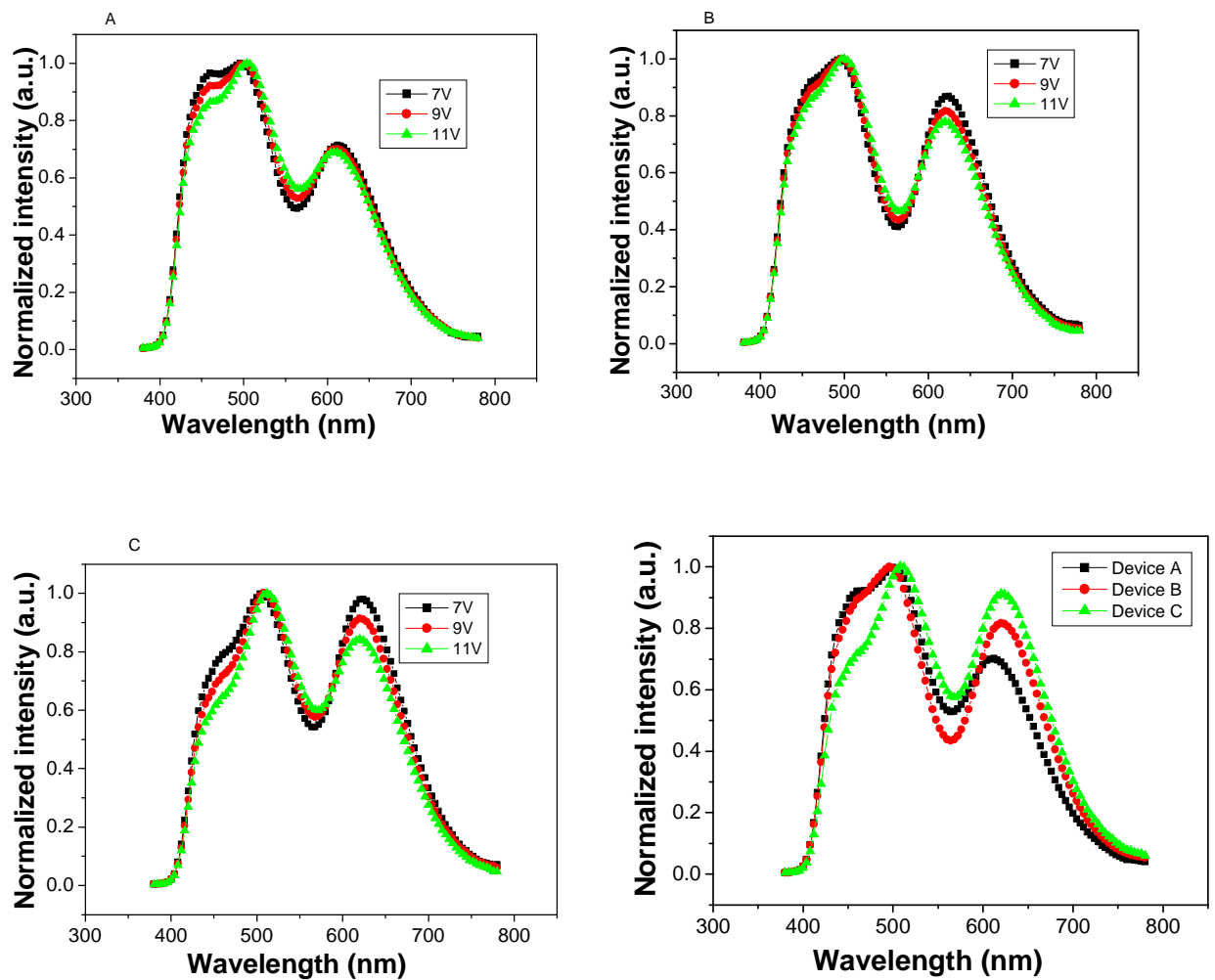

Fig. 2. Normalized EL intensity of the devices A-C at different voltage and Normalized EL intensity of devices A-C at $9 \mathrm{~V}$ 
Fig.3 shows the current density versus voltage $(\mathrm{J}-\mathrm{V})$ characteristics of the devices. With the increase thickness of sub-monolayer DCJTB, the current density for a given voltage decreases. The luminance-voltage characteristics of the devices are depicted in Fig.4, the device A and B have maximum luminance in same voltage. To be practical, an EL device should possess not only a high brightness but also high EL efficiency. For example, at a given voltage of $12 \mathrm{~V}$, the brightness of devices A-C is $6578 \mathrm{~cd} / \mathrm{m}^{2}, 6635 \mathrm{~cd} / \mathrm{m}^{2}$ and $2023 \mathrm{~cd} / \mathrm{m}^{2}$, respectively.

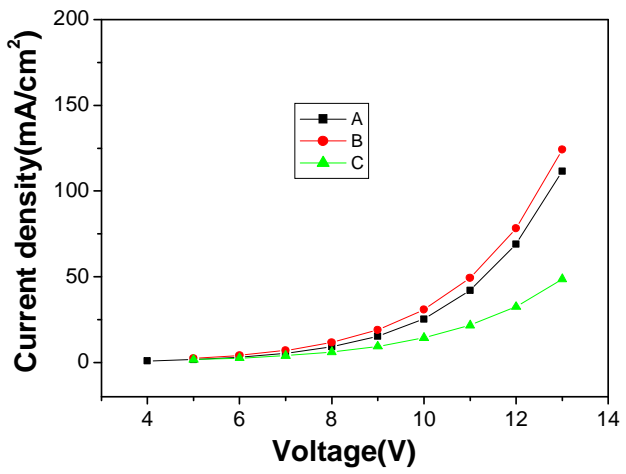

Fig 3.The current density versus voltage characteristics of the devices A-C

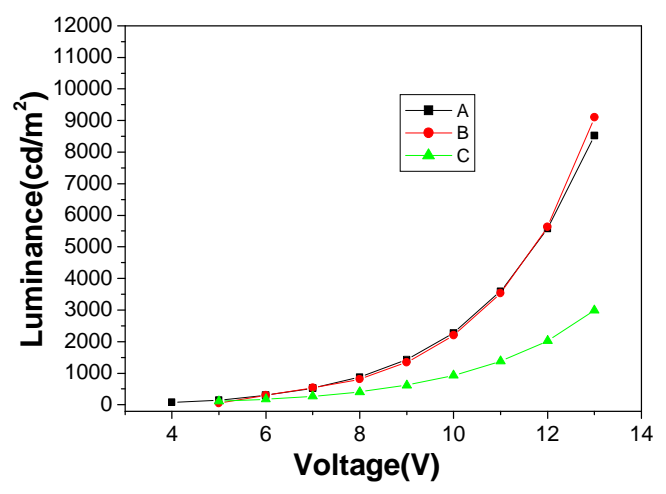

Fig 4. The luminance- voltage characteristics of devices A-C

Fig.5 shows the current efficiency-voltage of the devices. The maximum efficiency of devices A-C is $9.92 \mathrm{~cd} / \mathrm{A}$ at $6 \mathrm{~V}, 7.79 \mathrm{~cd} / \mathrm{A}$ at $7 \mathrm{~V}$ and $7.35 \mathrm{~cd} / \mathrm{A}$ at $5 \mathrm{~V}$, respectively. The device A exhibits the best current efficiency performance. The enhanced EL efficiency can be attributed to the perfect trapping in the red emitting layer. The current efficiency is comparatively depends on thickness of sub-monolayer DCJTB. The effect of concentration aggregation on the performance of organic emitting device has been invested previously. Our results indicates that for a given device structure, the proper sub-monolayer thickness can enhances the performance of organic emitting device.

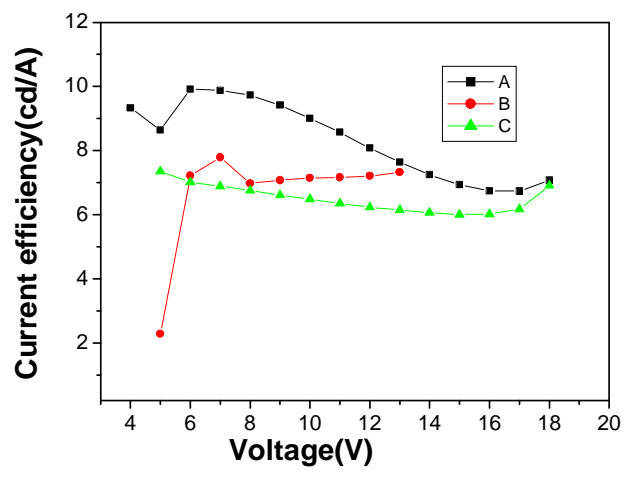

Fig 5. The current efficiency- voltage characteristics of devices A-D

Fig. 6 shows the optical and electronic characteristics of devices A-C, we can see that device A-C is white emit, the device A-C has the CIE coordinates change from $(0.3212,0.2992)$ at $4 \mathrm{~V}$ to $(0.3018$, $0.3185)$ at $13 \mathrm{~V},(0.3656,0.3919)$ at $5 \mathrm{~V}$ to $(0.3129,0.32)$ at $13 \mathrm{~V},(0.3474,0.3032)$ at $5 \mathrm{~V}$ to $(0.3393$, $0.3473)$ at $13 \mathrm{~V}$, respectively, that are well in the white region. The power current efficiency was 2.09 $\mathrm{lm} / \mathrm{W}$ at $4 \mathrm{~V}, 1.43 \mathrm{~lm} / \mathrm{W}$ at $7 \mathrm{~V}$ and $1.54 \mathrm{~lm} / \mathrm{W}$ at $5 \mathrm{~V}$, respectively. 

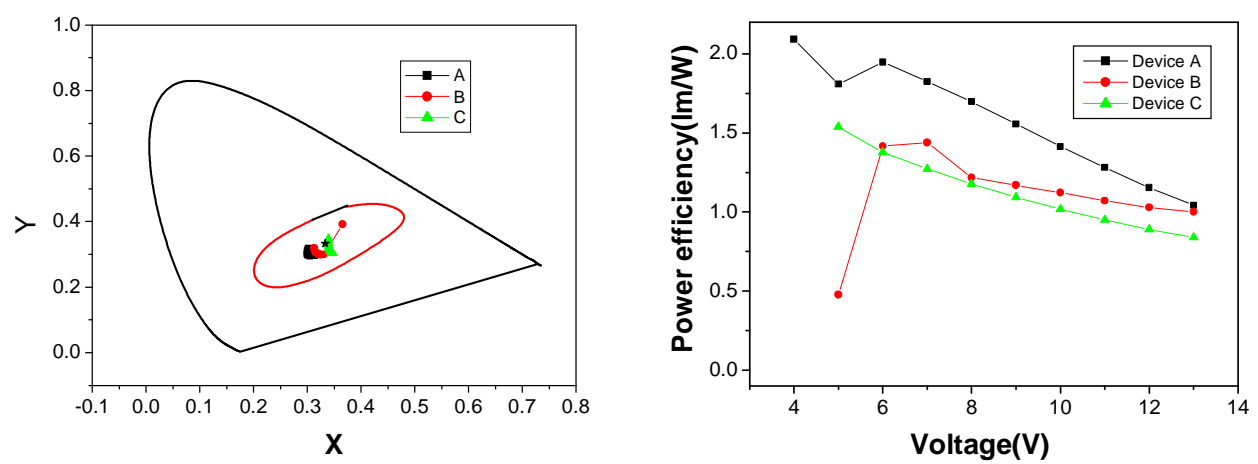

Fig6. The optical and electronic characteristics of devices A-C

\section{Summary}

We have fabricated efficient multilayer devices with blue fluorescent material combined with sub-monolayer red fluorescent dye and green dye. To change the thickness of sub-monolayer DCJTB, WOLEDs was obtained. When $\mathrm{x}=0.025$, the Commission Internationale de 1'Eclairage (CIE) coordinates of the device A change from $(0.3212,0.2992)$ at $4 \mathrm{~V}$ to $(0.3018,0.3185)$ at $13 \mathrm{~V}$ that are well in the white region, respectively. Its maximum luminance was $8520 \mathrm{~cd} / \mathrm{m}^{2}$ at $13 \mathrm{~V}$, maximum current efficiency, maximum power efficiency was $9.92 \mathrm{~cd} / \mathrm{A}$ at $6 \mathrm{~V}, 2.09 \mathrm{~lm} / \mathrm{W}$ at $4 \mathrm{~V}$, respectively.

\section{Acknowledgements}

This work was supported by the major project of science and technology office of Fujian Province of China (No.2014H0042), the Natural Science Foundation of Fujian Province of China (No. 2015J01664), and the project of Science and Technology Research of Quanzhou, Fujian Provice of China (No.2013Z125,2014Z137).

\section{References}

[1] C. W. Tang, S. A. VanSlyke:Appl. Phys. Lett, Vol. 51 (1987), p913

[2] Y. Han, L. Zhang, X. Zhang, K. Ruan, L. Cui, Y. Wang, L. Liao, Z. Wang and J. Jie: J. Mater. Chem. C, Vol.2(2014), p201.

[3] M. Kim, S. K. Jeon, S. H. Hwang and J. Y. Lee : Adv. Mater, Vol.27 (2015), p2515

[4] D. Zhang, L. Duan, Y. Zhang, M. Cai, D. Zhang and Y. Qiu : Light Sci. Appl, Vol.4(2015), pe232

[5] S. Wu, M. Aonuma, Q. Zhang, S. Huang, T. Nakagawa, K. Kuwabara and C. Adachi: J. Mater. Chem. C, Vol.2(2014), p421

[6] Y.Wang, S.H.Liu, F.Y.Dang, Y.Li, Y.M.Yin, J.Liu, K.Xu, X.C.Piao, W.F.Xie : J. Phys.D Appl.Phys, Vol.45(2012),p402002

[7] X.Piao, Y.Yin, J.Liu, Y.Li, K.Xu, Y.Wang, W.Xie : Org.Electron, Vol.13(2012),p2412

[8]Y.Yin,X.Piao,Y.Li,Y.Wang,J.Liu,K.Xu,W.Xie:Appl.Phys.Lett,Vol.101(2012),p063306

[9]M.Shao,X.Guo,S.F.Chen,Q.L.Fan,W.Huang : Chin.Phys.B, Vol.21(2012),p108507

[10]B. W. D’Andrade, R. J. Holmes, and S. R. Forrest: Adv. Mater,Vol.16(2004), p624

[11] Y. S. Wu, S. W. Hwang, H. H. Chen, M.T. Lee, W. J. Shen, C. H. Chen: Thin Solid Films, Vol. 488 (2005),p 265

[12] Xiaoming Wu, Liying Shen, Yulin Hua, Musen Dong, Yueju Su, Zhiqiang Jiao, Xiaoyan Yang, Shougen Yin: Journal of Luminescence, Vol.132(2012), p1261

[13] Chih-Hung Hsiao, Shun-Wei Liu, Chin-Ti Chen, Jiun-Haw Lee: Organic Electronics, Vol.11(2010),p1500 
[14] M. Strukeji, R. H. Jordan, A. Dodabalapur: J. Am. Chem. Soc., Vol. 118 (1996),p1213.

[15] Shixiong Liang, Zhaoxin Wu, Xuanke Zhao, Dawei Wang, Xun Hou, Zhijian Chen, Qihuang Gong: Optics Communications, Vol.285(2012),p1625 